\title{
A special pair of phytohormones controls excitability, slow closure, and external stomach formation in the Venus flytrap
}

\author{
María Escalante-Pérez ${ }^{a}$, Elzbieta Krol ${ }^{a}$, Annette Stange ${ }^{a}$, Dietmar Geiger ${ }^{a}$, Khaled A. S. Al-Rasheid ${ }^{b}$, Bettina Hause ${ }^{c}$, \\ Erwin Neher $^{\mathrm{d}, 1}$, and Rainer Hedrich ${ }^{\mathrm{a}, 1}$ \\ anstitute for Molecular Plant Physiology and Biophysics, University Wuerzburg, D-97070 Wuerzburg, Germany; ${ }^{b} Z o o l o g y$ Department, College of Science, \\ King Saud University, Riyadh 11451, Saudi Arabia; 'Department of Secondary Metabolism, Leibniz Institute of Plant Biochemistry, D-06120 Halle, Germany; \\ and ${ }^{\mathrm{d} D e p a r t m e n t}$ for Membrane Biophysics, Max Planck Institute for Biophysical Chemistry, D-37077 Goettingen, Germany
}

Contributed by Erwin Neher, August 2, 2011 (sent for review May 4, 2011)

Venus flytrap's leaves can catch an insect in a fraction of a second. Since the time of Charles Darwin, scientists have struggled to understand the sensory biology and biomechanics of this plant, Dionaea muscipula. Here we show that insect-capture of Dionaea traps is modulated by the phytohormone abscisic acid (ABA) and jasmonates. Water-stressed Dionaea, as well as those exposed to the drought-stress hormone $A B A$, are less sensitive to mechanical stimulation. In contrast, application of 12-oxo-phytodienoic acid (OPDA), a precursor of the phytohormone jasmonic acid (JA), the methyl ester of JA (Me-JA), and coronatine (COR), the molecular mimic of the isoleucine conjugate of JA (JA-lle), triggers secretion of digestive enzymes without any preceding mechanical stimulus. Such secretion is accompanied by slow trap closure. Under physiological conditions, insect-capture is associated with $\mathrm{Ca}^{2+}$ signaling and a rise in OPDA, Apparently, jasmonates bypass hapto-electric processes associated with trap closure. However, ABA does not affect OPDA-dependent gland activity. Therefore, signals for trap movement and secretion seem to involve separate pathways. Jasmonates are systemically active because application to a single trap induces secretion and slow closure not only in the given trap but also in all others. Furthermore, formerly touch-insensitive trap sectors are converted into mechanosensitive ones. These findings demonstrate that prey-catching Dionaea combines plant-specific signaling pathways, involving OPDA and ABA with a rapidly acting trigger, which uses ion channels, action potentials, and $\mathrm{Ca}^{2+}$ signals.

W hen a single trigger hair of the Venus flytrap (Dionaea muscipula) is bent twice or when two different sensory organs are bent once by an obstacle, the lobes of the trap close abruptly, "catching the prey." Insects touching these mechanosenory organs protruding from the upper leaf epidermis of the Venus flytrap activate mechanosensitive ion channels and generate receptor potentials, which induce an action potential (AP; refs. 1-3; for review, see refs. 4 and 5). A receptor potential always precedes an AP. Finally two APs couple the mechanical stimulation step to the release of the trap lobes accumulated elastic energy within $100 \mathrm{~ms}$ to shut the trap (initial fast closure) (6). However, the trap is not completely closed at this moment. To hermetically seal the trap, it requires ongoing activation of the mechanosensitive hairs by the trapped prey. Unless the prey is able to escape, it will further stimulate the inner surface of the lobes, thereby triggering further APs. This forces the edges of the lobes together, sealing the trap hermetically (prey-dependent slow closure) to form an external "stomach" in which prey digestion occurs. The second phase of trap closure is accompanied by secretion of lytic enzymes from the glands covering the inner surface of the bilobed leaf trap. Thus, digestive glands do not secrete until stimulated by natural or artificial prey (7). Additionally, prey-derived compounds stimulate digestive glands leading to acidification of the external stomach and production of lytic enzymes $(8,9)$. Although this type of plant carnivory has been known since Darwin's time, insights about the hapto-electrochemical coupling associated with the trapping behavior of Dio- naea remained rather limited. Many similarities between fast processes involved in carnivory and signals of the nervous system suggest similarity in mechanisms. Thus, ion channels and chemical factors are at the basis of triggering mechanisms in both systems (10). $\mathrm{Ca}^{2+}$ changes are involved in propagation and amplification of signals as well in triggering exocytosis, and secreted substances are important effectors in both cases. Here, we show that, in Dionaea, such signaling mechanisms are linked to plant-specific signal pathways involving a precursor of the phytohormone jasmonic acid (JA) as well as to the turgor-regulating phytohormone abscisic acid (ABA). Whereas the former systematically alerts neighboring traps to the presence of prey and elicits secretion, the latter regulates trap sensitivity, protecting the carnivore from untimely prey catching during periods of drought.

\section{Results}

ABA Counteracts Jasmonates by Suppressing Hapto-Electric Trap Contraction. ABA is a central element in turgor regulation within plant cells. Under drought stress, the carotene-derived sesquiterpene activates anion channels which leads e.g., to the down-regulation of guard cell turgor required for the closing movement of stomata. In the guard cells-operated system, JA/ coronatine (COR) affects the ABA-induced closing response (11). To study whether and how ABA interferes with fast trap closure in Dionaea, we sprayed the flytrap with a $50 \mu \mathrm{M}$ ABA solution. Following $48 \mathrm{~h}$ of $\mathrm{ABA}$ exposure, trigger hairs were mechanically stimulated. As in untreated traps, single trigger hair stimulations elicited typical APs; however, they did not elicit trap closure. Rather, at least three hair bendings were required to close the trap (Fig. 1A). Water stress, which is known to trigger ABA synthesis and signaling, impaired fast trap closure. To demonstrate this effect we interrupted irrigation and let the soil water content drop by $50-60 \%$. In this condition, more than two trigger hair stimulations were required to trigger fast trap closure (Fig. 1B). The loss of sensitivity was accompanied by an increase in ABA level in the trap lobes $(1,289 \pm 143 \mathrm{pmol} \mathrm{ABA} / \mathrm{gFW}$ in well watered Dionaea, and $3,460 \pm 936 \mathrm{pmol} \mathrm{ABA} / \mathrm{gFW}$ with water-deficient Dionaea at $50 \%$ soil water content, Fig. $1 C$ ). Thus, two distinct manipulations (exogenous application of ABA and drought stress) indicate an interaction between ABA signaling and the regulation of trap sensitivity.

Author contributions: M.E.-P., E.K., K.A.S.A.-R., E.N., and R.H. designed research; M.E.-P., E.K., A.S., and B.H. performed research; M.E.-P., E.K., A.S., D.G., B.H., E.N., and R.H. analyzed data; and M.E.-P., E.K., D.G., K.A.S.A.-R., B.H., E.N., and R.H. wrote the paper.

The authors declare no conflict of interest.

Freely available online through the PNAS open access option.

${ }^{1}$ To whom correspondence may be addressed. E-mail: eneher@gwdg.de or hedrich@ botanik.uni-wuerzburg.de.

This article contains supporting information online at www.pnas.org/lookup/suppl/doi:10 1073/pnas.1112535108/-/DCSupplemental. 

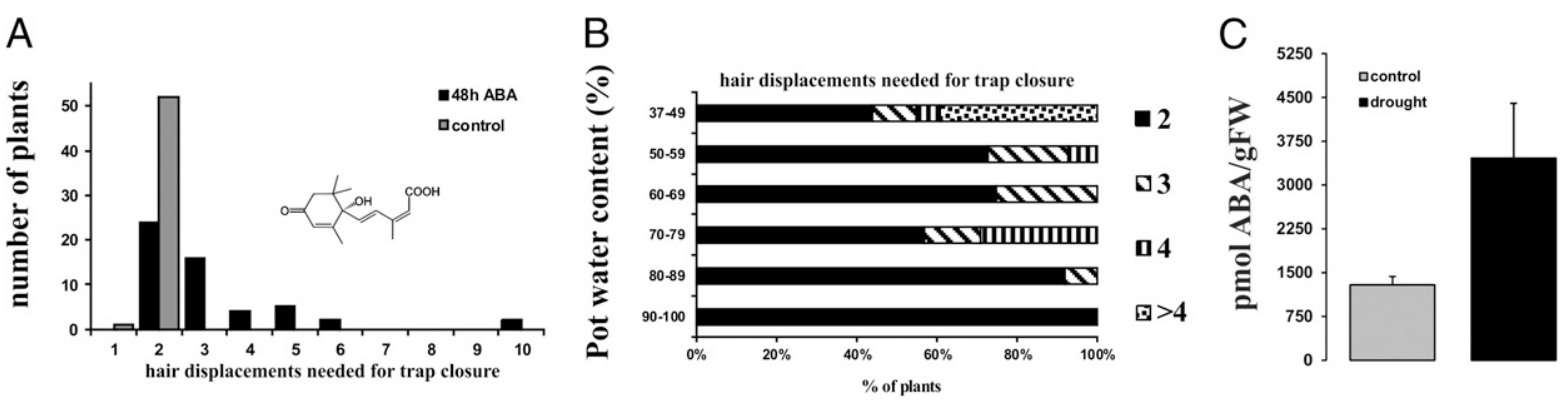

Fig. 1. (A) $\mathrm{ABA}(50 \mu \mathrm{M})$ desensitizes traps. Forty-eight hours after $A B A$ application, more than two trigger-hair displacements are required for trap closure $(n=60)$. (B) Upon drought stress (soil water content below $70 \%)$ more than two hair stimulations are required to elicit trap closure $(n=12)$. $(C)$ Quantification of $\mathrm{ABA}$ levels (pmol ABA/gFW) in the trap lobes of well watered and of water deficient Dionaea at $50 \%$ soil water content (FW, fresh weight, $n=6 \pm$ SD).

Long-Term Mechanical Stimulation Triggers 12-0xo-Phytodienoic Acid (OPDA) Synthesis. To study possible roles of "touch" hormones OPDA and JA, we exposed Dionaea plants to a colony of ants (Camponotus floridanus) and harvested traps, which had captured ants. OPDA and JA levels were analyzed and compared with those of traps, which had not been exposed to active prey. Thereby we observed a rise in OPDA level $30 \mathrm{~min}$ after the ants were captured (Fig. $2 A$ ). When open traps (without prey) were challenged with exogenous OPDA, COR, or Me-JA, they initially remained open, but their glands appeared to secrete fluids (Fig. 3). Upon application of COR, the Venus flytrap's performance became altered (Fig. 3 and Movie S1). (i) After 2 h $(2.6 \pm$ $0.45 \mathrm{~h}$ ), open traps started to open maximally, reaching a maximal opening after $6 \mathrm{~h}(6.2 \pm 0.24 \mathrm{~h})$. (ii) At that time, we observed changes in the light reflection; these changes are due to the secretion of fluids by the glands present in the inner surface of the trap. (iii) Upon secretion onset, slow trap closure set in $9 \mathrm{~h}$ after COR stimulation $(9 \pm 0.5 \mathrm{~h})$. (iv) Approximately $12 \mathrm{~h}(6 \mathrm{~h}$ plus $3.8 \pm 2.63 \mathrm{~h}$ ) after trap closure, external stomach formation was completed and the stomach was filled with an acidic fluid.

COR, known as phytotoxin secreted by several pathovars of the bacterial pathogen Pseudomonas syringae, exerts its virulence effects by co-opting the host's JA signaling pathway (12). To test whether COR, the 'prey substitute', causes exocytosis of digestive enzymes into the external stomach, we collected the secreted fluid humor in response to either ant capture or COR treatment. As a chemical trigger reference we used urea. Urea had already been shown by Darwin (7) to induce secretion in Dionaea. Following SDS/PAGE gel analysis, the protein pattern demonstrated that both a natural prey- and COR-triggered secretion with overlapping protein spectra and broad bands between 17 and $56 \mathrm{kDa}$ (Fig. S1). In contrast, urea-induced secretion resulted in two dominant protein bands (56 and $43 \mathrm{kDa}$ ) only, both of which were also found in the ant- and COR-derived fractions. This finding suggests that JA-related compounds trigger synthesis/exocytosis of lytic enzymes with spectra similar to those of the natural prey.

To quantify the COR-stimulated secretion, we counted the number of glands and determined the volume, protein content, and $\mathrm{pH}$ of secreted fluids over time. It became evident that the number of glands remained constant upon COR treatment (58 glands per $\mathrm{mm}^{2}, \sim 37,000$ glands per trap), but glands increased their volume/surface ratio. Untreated glands exhibited a constant volume/surface ratio (Fig. $4 B$ and $D$ ). The increase in the surface of glands appeared more pronounced at the basal region of the trap lobe compared with the outer parts of the lobe (Fig. $4 \mathrm{~A}$ and $D$ ). The volume and the total protein content of the secreted fluid increased with time after COR application in a dose dependent manner (Fig. $4 E$ and $F$ ). Whereas the volume of secreted fluid humor peaked after one week, the total protein content of the fluid increased constantly during two weeks of measurements. The increase in protein content was accompanied by an obvious increase in the viscosity of the secreted fluid.
Twelve hours after COR application the volume of secreted fluids enabled $\mathrm{pH}$ measurements. Monitoring proton extrusion revealed a pH of $\sim 4.3$ with steady state acidification (Fig. $4 G$ ). During the following 2 wk of fluid collection, the $\mathrm{pH}$ dropped down to 3.4 with traps treated either with $100 \mu \mathrm{M}$ or $1 \mathrm{mM}$ COR. Thus, OPDA, COR, and Me-JA are capable to mimic an edible prey in its role of eliciting secretion of digestive enzymes.

Jasmonates Systemically Induce Secretion in Open Traps. The lipidderived JA signal is produced in many plant species on sites of wounding and spreads, most likely via the phloem, to the nonwounded leaves $(13,14)$. To study whether the trap-associated $\mathrm{JA}$ response is also systemic in nature, we stimulated only one single trap of Dionaea plants with jasmonates. Such stimulation induced secretion of all other neighboring traps. Glands of younger traps became active before the older ones, independent of the site where OPDA/Me-JA/COR was applied. In line with the suggested transmission of jasmonate-related compounds via the phloem, stimulated source traps induced all other traps in a manner dependent on sink strength (15). These compounds thus seem to function as systemic secretion inductors. Interestingly, following COR-only treatment, OPDA levels increased locally and in a systemic manner (Fig. $4 H$ ). Because JA biosynthesis is known to be regulated by positive feedback (16), this response points to an induction of OPDA/JA biosynthesis by COR. Our data show that jasmonates, which in most plants systemically signal the presence of a predator, also have a role in the systemic regulating of trap responses.

Two APs Close the Trap, Three APs Activate Glands. Bending of trigger hairs elicits a 1-s-long AP that initiates at the base of the multicellular sensory structure in one lobe and spreads over the entire trap to the opposite lobe (17-20). It is generally accepted that the resting potential in the flytrap is stabilized by a plasma

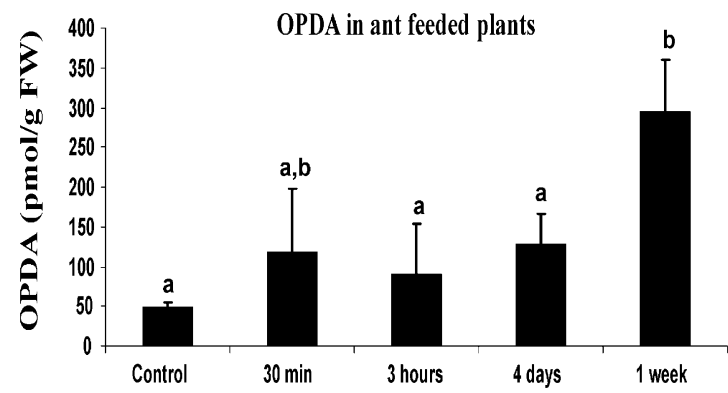

Fig. 2. (A) Insect (ant) capture results in OPDA synthesis. Note that OPDA levels increased already more than twofold $30 \mathrm{~min}$ after prey capture. Maximal levels (sixfold) were measured after $1 \mathrm{wk}(n=4$, one-way ANOVA, Tukey HSD test, $P<0.05$ ) 

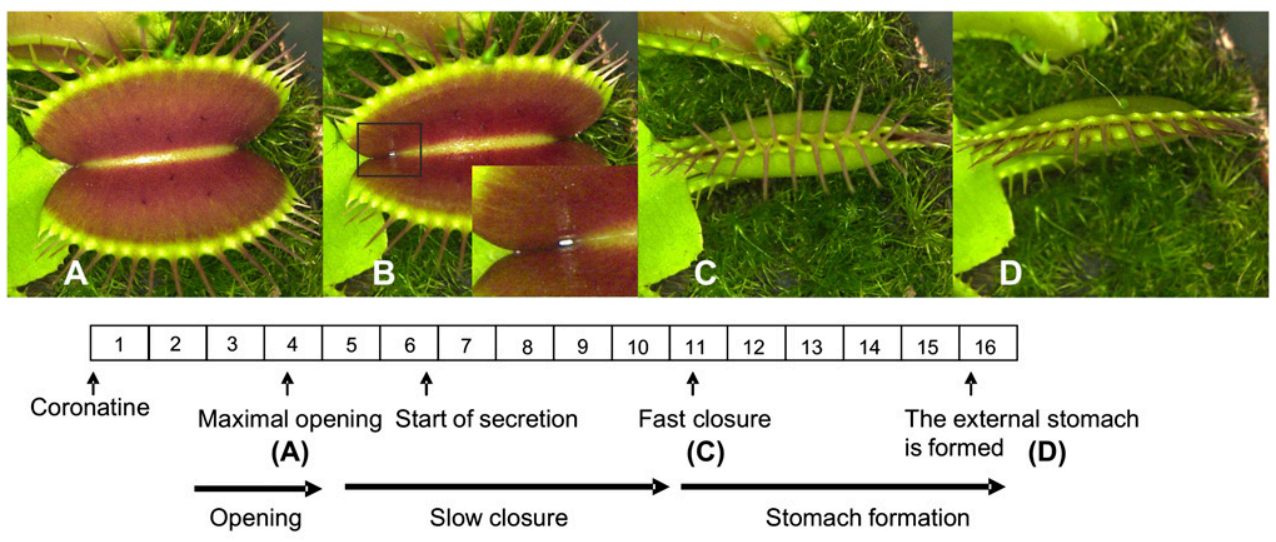

Secretion (B)

Fig. 3. COR mimics an eatable prey. Spray application of Dionaea lobes with $1 \mathrm{mM}$ COR induced transient opening of the trap, followed by secretion onset, slow trap closure, and external stomach formation. Time (in hours) is shown inside the boxes $(n=5)$. membrane potassium conductance. During the AP, a calciumactivated, time-dependent chloride conductance provides for the transient membrane depolarization (21). So far, nothing is known about whether and how mechano-electric stimulation is recognized by the secretory system of the trap. To study the membrane response of the secretory cells, we impaled the first or second cell layer of the gland with microelectrodes (Fig. 5A). Following electrode insertion into a nontreated cell, a resting potential of $-115 \pm 8 \mathrm{mV}(n=10)$ was recorded. When we mechanically stimulated the trigger hair, an AP was fired (Fig. $5 B$ ), but touching other trap lobe cells of the inner or outer trap lobe did not elicit transient membrane potential changes. Trigger hair displacements, however, resulted in APs in the Dionaea gland cells that were similar in amplitude $(131 \pm 15 \mathrm{mV})$ and kinetics $\left(t_{1 / 2}=0.3 \pm 0.06 \mathrm{~s}\right)$ to that of other trap lobe cells (17).

To further study the events in the Dionaea gland triggered by sensory hair displacements, we excised individual trap lobes and placed them in a recording chamber. This approach enabled us to record the electrical behavior of secretory cells unperturbed by trap movement. Following impalement by double-barreled microelectrodes, gland cells were iontophoretically loaded with the calcium indicator dye FURA-2. Due to the symplastic connection between the gland cells, FURA-2 injected into a single cell spread over the entire gland cell complex (Fig. $5 A$ Inset). In this situation, single and double stimulation of the sensory hairs elicited APs without a measurable increase in cytosolic calcium concentration. More than two APs, however, elicited single calcium transients or even $\left[\mathrm{Ca}^{2+}\right]$ oscillations (Fig. $5 E$ and Fig. S2). These findings confirm the notion that AP peak depolarization is associated with plasma membrane calcium fluxes $(17,21)$. In addition, they suggest that more than two APs are required to elicit a cytosolic calcium signal within the gland cell bodies. Based on these results, we conclude that the insects' attempts to break out of a trap result in repetitive hair stimulation in glands inducing trains of APs, a rise in the cytosolic calcium, and, in turn, a rise calcium-induced secretion.

Traps incubated with COR 8-12 h before effector application remained electrically silent. When we induced APs by trigger hair displacements, the AP appeared reduced in amplitude by $31 \%(90 \pm 19 \mathrm{mV})$ and the AP duration (measured at $50 \%$ of amplitude, $t_{1 / 2}$, see Fig. $5 C$ Inset $)$ was prolonged by $62 \%(0.5 \pm$ $0.09 \mathrm{~s})$ compared with those not having seen the signaling compound before (Fig. $5 B$ and $C$ ). The influence of COR on the size of the AP is reminiscent to that recorded with Dionaea traps under reduced extracellular calcium concentration (18). Interestingly, however, spontaneous firing of APs can also be observed after COR-stimulated secretion onset (Fig. 5D).

To test whether a touch-induced rise of jasmonates is altering the trapping behavior, we mechanically stimulated trigger hairs and the outer trap lobe. After such treatments, a single dis- placement of just one sensory hair sometimes elicited two APs or more. This result indicates that the threshold to induce APs was lowered. In line with this hypothesis, simply touching the outer trap lobe occasionally resulted in closure. The lipid-derived signals thus seem to increase touch sensitivity and to reduce mechanical stability of the open trap.

\section{Discussion}

Among the various lipid-derived signaling molecules in plants, jasmonates and octadecanoids are known to function in response to abiotic and biotic stresses as well as in development. They regulate induced defense mechanisms in plants after insect attack and during the wound response in general (16). A characteristic feature of the wound response is an endogenous rise in OPDA, JA, and its metabolites, followed by local and systemic expression of JA-induced genes $(14,16,22)$. Jasmonates, such as JA and its various metabolites, are synthesized via the octadecanoid pathway, where OPDA is a central intermediate (23) However, OPDA is of special importance due to its JA-independent signaling properties in response of plants to pathogens and touch $(24,25)$.

A behavior related to the slow closure of D. muscipula is known from Bryonia dioica, where mechano-stimulation triggers the curvature of tendrils (26). This process can be elicited also by the airborne methyl ester of JA without any mechanical contact (27). In this way, the chemically induced reaction is morphologically and biochemically indistinguishable from the mechanically induced process. Interestingly, in tendril coiling, the endogenous signal is OPDA, but not JA (28). The OPDA level rises transiently and correlates with the degree of coiling, whereas JA levels remain low and constant (29). Furthermore, structure activity tests with numerous structural derivatives of jasmonates revealed clear preference for octadecanoid-like compounds as inducers of tendril coiling (30). In addition, treatment with COR can elicit physiological changes reminiscent of thigmomorphogenesis (29). However, COR and JA are active only indirectly by increasing the endogenous level of OPDA (31).

Here, we show that Dionaea uses these plant-specific pathways for regulating a cascade of events subsequent to prey catching. COR treatment mimics an edible prey, leading to trap closure and secretion by the glands, even without mechanical stimulation (Fig. 3). The secreted protein pattern appeared identical to that of secreted proteins upon insect capture (Fig. S1). Furthermore, we demonstrate by electrophysiological measurements that COR-treated plants are systematically sensitized, responding with spontaneous APs without being triggered by the mechanosensitive hairs (Fig. 5D). Stimulation of trigger hairs of CORtreated traps resulted in prolonged APs with a reduced amplitude compared with untreated controls (Fig. $5 B$ ). This behavior 

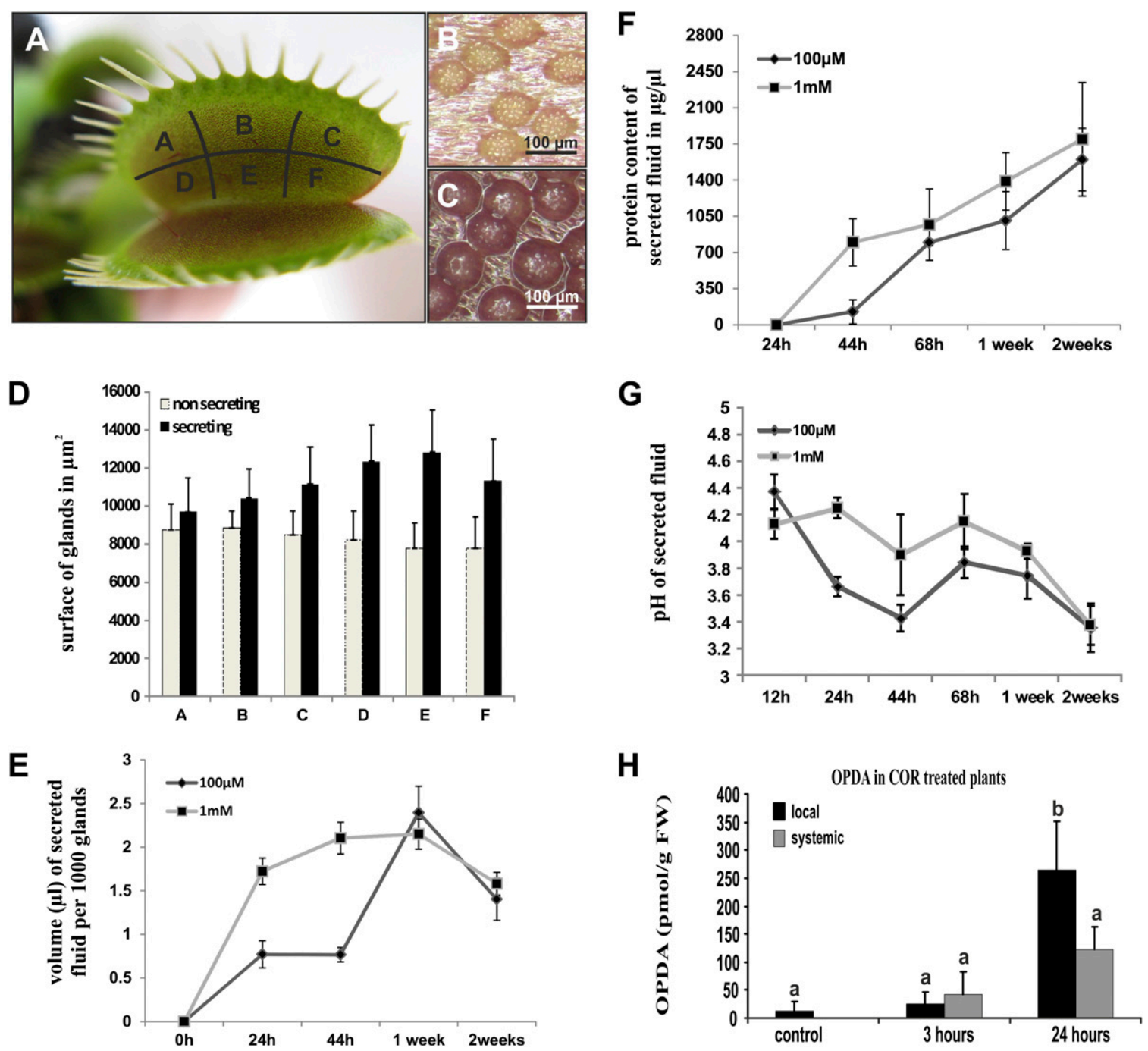

Fig. 4. Quantification of COR-induced secretion. ( $A$ ) The lobe of a Dionaea trap was divided in six sectors (A to $F$ ), and the number of glands were counted. ( $B$ and $C$ ) Close-up view of nonsecreting glands $(B)$ in comparison with COR-stimulated secreting glands $(C)$. $(D)$ Quantification of gland surfaces of nonsecreting and COR-stimulated ( $48 \mathrm{~h}, 100 \mu \mathrm{M}$ COR) secreting Dionaea traps divided into sectors as illustrated in $A(n=20$ traps \pm SD). (E-G) Quantification of the volume $(E, n=10 \pm \mathrm{SE})$, protein content $(F, n=6 \pm \mathrm{SE})$, and $\mathrm{pH}(G, n=12 \pm \mathrm{SE})$ of secreted fluids plotted against the time after COR application. (H) Exogenous application of $1 \mathrm{mM}$ COR increased endogenous OPDA levels in COR-treated traps (local) as well as in nonstimulated traps (systemic; $n=4$, one-way ANOVA, Tukey HSD test, $P<0.05$ ).

might alert the remaining open traps of a Dionaea plant. Insects such as ants often come in colonies. The successful capture of one insect leads to the production of OPDA, which is delivered to the other traps in a systemic fashion (Fig. 2). Thus, OPDA or an OPDA-related compound might carry the message 'where there is one, there might soon be more'.

Simultaneous measurements of APs and cytosolic $\mathrm{Ca}^{2+}$ signals revealed, that two APs, which elicit trap closure (32) are not sufficient for triggering a detectable cytosolic $\mathrm{Ca}^{2+}$ increase. Interestingly a third AP resulted in the detectable rise of $\left[\mathrm{Ca}^{2+}\right]_{\mathrm{cyt}}$ (Fig. 5E and Fig. S2). Already Hodick and Sievers (18) proposed that the elevation of $\left[\mathrm{Ca}^{2+}\right]_{\text {cyt }}$ may be an important factor in the "memory" of the trap. Our results suggest that the attempt of insects to escape the trap leads to repetitive AP triggering and thereby induces a rise in the cytosolic calcium. Thus, the elevation of calcium might be an important second messenger for stomach formation and gland secretion rather than for fast trap closure. Note that calcium triggered secretion in plants has already been described for the case of hydrolytic enzyme-secreting aleuron cells (33).
It is well known that the drought-stress hormone ABA affects tugor-driven processes in all plants (34). We found that trap does not work when Dionaea is not properly irrigated and soil water content drops (Fig. 1). We also showed that the ABA level of Dionaea increases under such conditions. It has been shown that $\mathrm{ABA}$ and mechano-stimulation at least partially share signaling networks (35). ABA is synthesized when plants experience drought (36), but to digest a prey, the Venus flytrap has to invest an enormous amount of water. This investment very likely is lethal with progressive soil drying. Our demonstration that $\mathrm{ABA}$ reduces the touch sensitivity of Dionaea suggests that the plant balances the gain of nitrogen- and carbon-rich food against the water consumption, needed to digest a prey. This "water use efficiency" strategy is based on ABA signaling (37), well known from noncarnivorous plants. In general, drought stress of plants causes the delivery of root-produced ABA to leaves, to prevent guard cell swelling and the opening movement of the stomata. As a result, the $\mathrm{CO}_{2}$ uptake and carbon gain via photosynthesis is inhibited (i.e., the plant gets no food). On the other hand, transpiration is largely prevented (i.e., the plant saves water). 

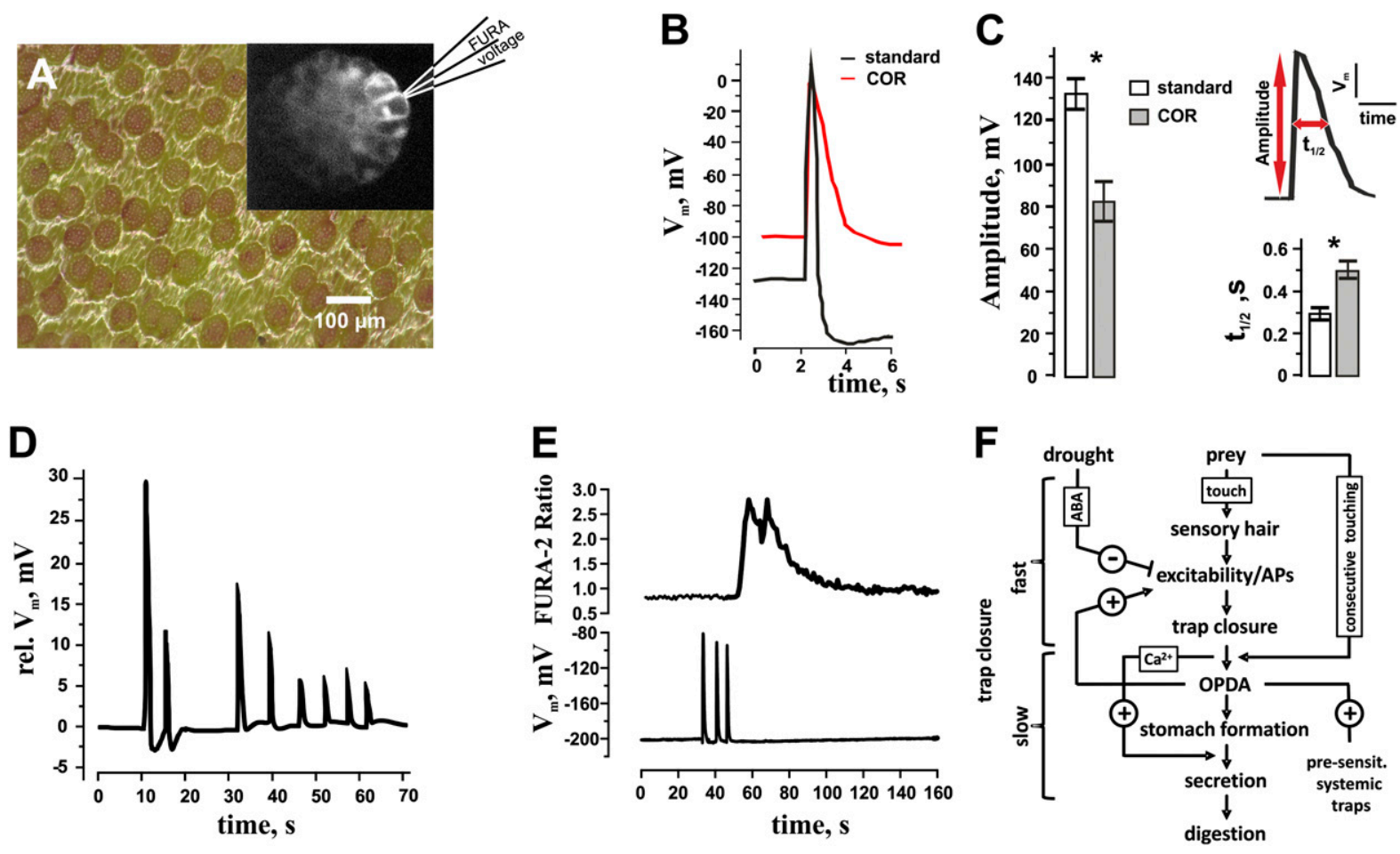

Fig. 5. (A) View on the inner leaflet of a trap lobe. (Inset) FURA-2 loaded iontophoretically into a gland cell complex via a micropipette impaled into a secretory cell. (B) AP in responses to single trigger hair displacements in the presence (red) and absence (black) of 1 mM COR. (C) Quantification of APs shown in A. (Inset) AP amplitude (Upper) and duration (at $50 \%$ of amplitude, Lower). The columns represent the average of at least six independent measurements; the bars are \pm SD ( $t$ test, $P \leq 0.001$ indicated by an asterisk). $(D)$ Spontaneous APs (relative values) leading to a trap closure $8.5 \mathrm{~h}$ after COR exposure. Four of the six examined plants generated a series of APs after trap closure. ( $E$ ) Calcium transient elicited by 3 APs. APs were elicted by individual mechanical stimulation of single trigger hairs. A Dionaea gland cell was impaled with a double-barrelled microelectrode and iontophoretically loaded with the calcium sensitive dye FURA-2 as shown in A Inset. Plasma-membrane potential (lower trace) monitored simultaneously with the FURA-2 F345/390 fluorescence ratio (upper trace). In 7 of 14 cells, the AP was accompanied by a calcium transient. ( $F$ ) Scheme for the involvement of ABA and jasmonates (OPDA) in the prey capture by $D$. muscipula. When trigger hairs of the Venus flytrap are mechanically stimulated by a prey, mechanosensitive ion channels are activated and generate receptor potentials, which in turn induce APs and the lobes of the trap abruptly close. A successful prey capture leads to consecutive touching of the trigger hairs by the struggling prey, which stimulates the synthesis of OPDA and the rise of cytosolic [Ca ${ }^{2+}$. These chemical factors herald the start of the second phase of trap closure, which is accompanied by secretion of lytic enzymes from the glands and the hermetically sealing of the trap (stomach formation). In addition, the synthesis of OPDA systemically presensitizes other traps of the carnivore and positively feeds back on trap excitability. In contrast, drought stress (ABA) decreases the excitability of traps because prey digestion is accompanied by an enormous consumption of water.

Recently the fast ABA-signaling pathway leading to anionchannel-induced stomatal closure could be reconstituted $(38,39)$. The basic/fast signal transduction chain consists of only four components in Arabidopsis: (i) A cytosolic ABA receptor (PYR/ PYL/RCAR-family $(40,41)$ controls directly the activity of (ii) PP2C protein phosphatases (e.g., ABI1/2 or HAB1/2) in an ABAdependent manner. Upon ABA binding, the phosphatases are inhibited and in turn downstream (iii) kinases of the SnRK2 (OST1) or SnRK3 (CPK) protein kinase families are released from blockage. (iv) SLAC1, a guard cell S-type anion channel that resembles the target of these kinases, is activated upon phosphorylation. Finally, the release of anions causes stomatal closure. In contrast to these fast responses, the long-term droughtinduced desensitization of the flytrap's capture apparatus is most likely transcriptionally regulated. In Arabidopsis, the fast and the slow transcriptional adaption to drought stress share, for the most part, the same ABA-signaling components $(38,42)$. Both pathways are controlled by PYR/PYL/RCAR ABA receptors, PP2c phosphatases, and protein kinases of the SnRK2 family. Genome/ transcriptome analysis of carnivorous plants combined with functional expression in heterologous systems should elucidate the evolutionary similarities and discrepancies in drought (stress) adaption between these different plant families.

Our results imply that phosphorylation/dephosphorylation reactions are important modulators of plant carnivore excitability. This reveals another analogy to the nervous system, where both electrical excitability and synaptic transmission is strongly modulated by such posttranslational modifications. Dionaea assembles an extensive signaling network that relies partly on plant-specific components (which it uses for its own specific purposes) and partly on mechanisms resembling those of higher animals. However, in contrast to nerve cells in animals, terrestrial plants lack fast voltage-dependent $\mathrm{Na}^{+}$channels, possibly due to the fact that $\mathrm{Na}^{+}$gradients in such plants are minor. Thus, $\mathrm{Na}^{+}$currents would not be very efficient to transiently depolarize the membrane potential. Instead, plants possess a rapid (R-type) anion channel current component in addition to the slow (S-type) anion channels (SLAC1). This anion channel exhibits voltage-dependent features of neuronal calcium and sodium channels (4346). Upon depolarization, this channel type activates with fast kinetics, whereas hyperpolarization causes deactivation. Hence, this type of plant anion channel has all of the properties to substitute for $\mathrm{Na}^{+}$channels and to drive the Dionaea AP.

Sequencing the genome of the Venus flytrap and identifying the genes encoding key elements in mechanoelectric trap contraction will allow us to further understand the action of the Darwin plant and to characterize both similarities and differences between analogous processes in the two kingdoms. Furthermore, with the ion channel genes identified and functionally expressed $(39,47)$, it will be possible to reconstitute the Dionaea $\mathrm{AP}$ and secretion process known since Darwin's time (48). 


\section{Methods}

Plants. D. muscipula plants were purchased from CRESCO Carnivora and grown in plastic pots at $26{ }^{\circ} \mathrm{C}$ with $12: 12 \mathrm{~h}$ light:dark photoperiod. Intact plants were sprayed with coronatine/Me-Ja/OPDA solution containing $0.01 \%$ Tween.

OPDA Quantification. OPDA and JA were extracted from plant material and analyzed according to Hause et al. (49). Fractions containing OPDA and JA were collected separately, derivatized, and analyzed by GC-MS according to Miersch et al. (22).

Quantitative Measurements. Secretion was stimulated by inoculation with 1 $\mathrm{cm}^{2}$ ashless (Whatmans' $\mathrm{n}^{\circ}$ 451) filter paper strips loaded with $100 \mu$ l of either $100 \mu \mathrm{M}$ or $1 \mathrm{mM}$ coronatine. After different time points, the filter paper was centrifuged and the different parameters were analyzed. For further information, see SI Methods.

Intracellular Measurements. Before measurements, the lobe of a cut trap was glued to a chamber bottom and left for recovery for at least $0.5 \mathrm{~h}$ in a standard solution containing $0.1 \mathrm{mM} \mathrm{KCl}, 10 \mathrm{mM} \mathrm{CaCl}_{2}$, and $5 \mathrm{mM}$ Mes adjusted with Tris to $\mathrm{pH} 6$. In the course of experiments, leaves were constantly perfused with the standard solution (1 $\mathrm{mL} / \mathrm{min})$. For details, see $\mathrm{S} /$ Methods.

1. Burdon-Sanderson J (1874) Venus fly-trap (Dionaea muscipula). Nature 10:105-107, 127-128.

2. Burdon-Sanderson J (1882) On the electromotive properties of the leaf of Dionaea in the excited and unexcited states. Philos Trans R Soc Lond B Biol Sci 173:1-55.

3. Burdon-Sanderson J, Page $F$ (1876) On the mechanical effects and on the electrical disturbance consequent on excitation of the leaf of Dionaea muscipula. Philos Trans $R$ Soc Lond B Biol Sci 25:411-434.

4. Volkov AG, Adesina T, Jovanov E (2007) Closing of venus flytrap by electrical stimulation of motor cells. Plant Signal Behav 2:139-145.

5. Volkov AG, Adesina T, Markin VS, Jovanov E (2008) Kinetics and mechanism of Dionaea muscipula trap closing. Plant Physiol 146:694-702.

6. Forterre Y, Skotheim JM, Dumais J, Mahadevan L (2005) How the Venus flytrap snaps. Nature 433:421-425.

7. Darwin CR (1875) Isectivorous plants (D. Appleton and Company, New York).

8. Rea PA (1983) The dynamics of $\mathrm{H}^{+}$efflux from the trap lobes of Dionaea-muscipula (Venus-flytrap). Plant Cell Environ 6:125-134.

9. Scala J, lott K, Schwab DW, Semersky FE (1969) Digestive Secretion of Dionaea muscipula (Venus's Flytrap). Plant Physiol 44:367-371.

10. Ueda $\mathrm{M}$, et al. (2010) Trap-closing chemical factors of the Venus flytrap (Dionaea muscipulla Ellis). ChemBioChem 11:2378-2383.

11. Munemasa S, et al. (2007) The coronatine-insensitive 1 mutation reveals the hormona signaling interaction between abscisic acid and methyl jasmonate in Arabidopsis guard cells. Specific impairment of ion channel activation and second messenger production. Plant Physiol 143:1398-1407.

12. Brooks DM, Bender CL, Kunkel BN (2005) The Pseudomonas syringae phytotoxin coronatine promotes virulence by overcoming salicylic acid-dependent defences in Arabidopsis thaliana. Mol Plant Pathol 6:629-639.

13. Glauser G, et al. (2009) Velocity estimates for signal propagation leading to systemic jasmonic acid accumulation in wounded Arabidopsis. J Biol Chem 284:34506-34513.

14. Koo AJK, Howe GA (2009) The wound hormone jasmonate. Phytochemistry 70 $1571-1580$

15. Thorpe MR, Ferrieri AP, Herth MM, Ferrieri RA (2007) 11C-imaging: methyl jasmonate moves in both phloem and xylem, promotes transport of jasmonate, and of photoassimilate even after proton transport is decoupled. Planta 226:541-551.

16. Wasternack C (2007) Jasmonates: an update on biosynthesis, signal transduction and action in plant stress response, growth and development. Ann Bot (Lond) 100:681-697.

17. Hodick D, Sievers A (1986) The influence of $\mathrm{Ca}^{2+}$ on the action-potential in mesophyllcells of Dionaea muscipula Ellis. Protoplasma 133:83-84.

18. Hodick D, Sievers A (1988) The action-potential of Dionaea-muscipula Ellis. Planta 174:8-18

19. Jacobson SL (1965) Receptor response in Venus's fly-trap. J Gen Physio/ 49:117-129.

20. Sibaoka T (1966) Action potentials in plant organs. Symp Soc Exp Biol 20:49-73.

21. Fromm J, Lautner S (2007) Electrical signals and their physiological significance in plants. Plant Cell Environ 30:249-257.

22. Miersch O, Neumerkel J, Dippe M, Stenzel I, Wasternack C (2008) Hydroxylated jasmonates are commonly occurring metabolites of jasmonic acid and contribute to a partial switch-off in jasmonate signaling. New Phytol 177:114-127.

23. Feussner I, Wasternack C (2002) The lipoxygenase pathway. Annu Rev Plant Biol 53: 275-297.

24. Chehab EW, Eich E, Braam J (2009) Thigmomorphogenesis: a complex plant response to mechano-stimulation. J Exp Bot 60:43-56.

25. Mueller MJ (1997) Enzymes involved in jasmonic acid biosynthesis. Physiol Plant 100: 653-663.

26. Weiler E (1997) Grundlagen der pflanzlichen Mechanosensorik. Naturw. Rdsch. 50: 337-342.

27. Falkenstein E, Groth B, Mithöfer A, Weiler E (1991) Methyljasmonate and alpha-linolenic acid are potent inducers of tendril coiling. Planta 185:316-322.
Calcium Measurements. Before measurements, lobes of dissected traps were mounted to a recording chamber and exposed for $0.5 \mathrm{~h}$ to a buffer containing $0.1 \mathrm{mM} \mathrm{KCl}, 10 \mathrm{mM} \mathrm{CaCl}_{2}, 20 \mathrm{mM}$ sorbitol, and $5 \mathrm{mM}$ Mes adjusted with Tris to $\mathrm{pH}$ 6. Microelectrode fabrication and recordings as well as FURA-2 based calcium monitoring was performed according to Levchenko et al. (50), using the same instrumentation.

ABA Measurements. Ground tissue samples were homogenized and extracted in $80 \%$ aqueous methanol. Extracts were passed through a Sep Pak $C_{18}$ cartridge. Methanol was removed under reduced pressure, and the aqueous residue was partitioned three times against ethyl acetate at $\mathrm{pH}$ 3.0. The ethyl acetate of the combined organic fractions was removed under reduced pressure. The newly obtained residue was resuspended in Tris-buffered saline (TBS; $150 \mathrm{mmol} / \mathrm{l} \mathrm{NaCl} 1 \mathrm{mmol} / \mathrm{L} \mathrm{MgCl}$, and $50 \mathrm{mmol} / \mathrm{L}$ Tris at $\mathrm{pH} \mathrm{7.8)}$ and subjected to an immunological ABA assay (ELISA) as described $(51,52)$.

ACKNOWLEDGMENTS. We thank Gregory Harms and Thomas Müller for critical reading and helpful comments on the manuscript. We thank Hagen Stellmach and Thomas Hofmann for excellent technical assistance. Sincere thanks to Enrique Lopez-Sanjurjo for help with protein gels and to Prof. Roces (University of Würzburg) for providing the ants for the experiments. This work was supported by a King Saud University grant (to R.H., E.N., and K.A.S.A.-R.), by Deutsche Forschungsgemeinschaft grants within GK1342 (to A.S.), and by an Alexander von Humboldt stipend (to E.K.).

28. Weiler EW, et al. (1994) The Pseudomonas phytotoxin coronatine mimics octadecanoid signalling molecules of higher plants. FEBS Lett 345:9-13.

29. Stelmach BA, et al. (1998) Quantitation of the octadecanoid 12-oxo-phytodienoic acid, a signalling compound in plant mechanotransduction. Phytochemistry Special Issue in Honor of Professor Clarence A. Ryan 47:539-546.

30. Blechert $\mathrm{S}$, et al. (1999) Structure-activity analyses reveal the existence of two separate groups of active octadecanoids in elicitation of the tendril-coiling response of Bryonia dioica Jacq. Planta 207:470-479.

31. Stelmach BA, Müller A, Weiler EW (1999) 12-Oxo-phytodienoic acid and indole-3-acetic acid in jasmonic acid-treated tendrils of Bryonia dioica. Phytochemistry 51:187-192.

32. Brown WH (1916) The mechanism of movement and the duration of the effect of stimulation in the leaves of Dionaea. Am J Bot 3:68-90.

33. Tester M, Zorec R (1992) Cytoplasmic calcium stimulates exocytosis in a plant secretory cell. Biophys J 63:864-867.

34. Jones H, Leigh RA, Tomos AD, Jones RGW (1987) The effect of abscisic-acid on cell turgor pressures, solute content and growth of wheat roots. Planta 170:257-262.

35. Delk NA, Johnson KA, Chowdhury NI, Braam J (2005) CML24, regulated in expression by diverse stimuli, encodes a potential $\mathrm{Ca}^{2+}$ sensor that functions in responses to abscisic acid, daylength, and ion stress. Plant Physiol 139:240-253.

36. Beck EH, Fettig S, Knake C, Hartig K, Bhattarai T (2007) Specific and unspecific responses of plants to cold and drought stress. J Biosci 32:501-510.

37. Zhang JH, Jia WS, Yang JC, Ismail AM (2006) Role of ABA in integrating plant responses to drought and salt stresses. Field Crops Res 97:111-119.

38. Geiger D, et al. (2010) Guard cell anion channel SLAC1 is regulated by CDPK protein kinases with distinct $\mathrm{Ca}^{2+}$ affinities. Proc Natl Acad Sci USA 107:8023-8028.

39. Geiger D, et al. (2009) Activity of guard cell anion channel SLAC1 is controlled by droughtstress signaling kinase-phosphatase pair. Proc Natl Acad Sci USA 106:21425-21430.

40. Ma Y, et al. (2009) Regulators of PP2C phosphatase activity function as abscisic acid sensors. Science 324:1064-1068.

41. Park SY, et al. (2009) Abscisic acid inhibits type $2 \mathrm{C}$ protein phosphatases via the PYR/ PYL family of START proteins. Science 324:1068-1071.

42. Fujii $\mathrm{H}$, et al. (2009) In vitro reconstitution of an abscisic acid signalling pathway. Nature 462:660-664.

43. Hedrich $\mathrm{R}$, Busch $\mathrm{H}$, Raschke $\mathrm{K}(1990) \mathrm{Ca}^{2+}$ and nucleotide dependent regulation of voltage dependent anion channels in the plasma membrane of guard cells. EMBO J 9:3889-3892.

44. Hille B (2001) Ionic Channels of Excitable Membranes (Sinauer, Sunderland, MA), 3rd Ed.

45. Kolb HA, Marten I, Hedrich R (1995) Hodgkin-Huxley analysis of a GCAC1 anion channel in the plasma membrane of guard cells. J Membr Biol 146:273-282.

46. Meyer S, et al. (2010) AtALMT12 represents an R-type anion channel required for stomatal movement in Arabidopsis guard cells. Plant J 63:1054-1062.

47. Ivashikina N, et al. (2001) $\left.\mathrm{K}^{+}\right)$channel profile and electrical properties of Arabidopsis root hairs. FEBS Lett 508:463-469.

48. Burdon-Sanderson J (1873) Note on the electrical phenomena which accompany irritation of the leaf of Dionaea muscipula (Venus flytrap). Proc. R. Soc. 21:495-496.

49. Hause B, Maier W, Miersch O, Kramell R, Strack D (2002) Induction of jasmonate biosynthesis in arbuscular mycorrhizal barley roots. Plant Physiol 130:1213-1220.

50. Levchenko V, Konrad KR, Dietrich P, Roelfsema MRG, Hedrich R (2005) Cytosolic abscisic acid activates guard cell anion channels without preceding $\mathrm{Ca}^{2+}$ signals. Proc Natl Acad Sci USA 102:4203-4208.

51. Mertens R, Deusneumann B, Weiler EW (1983) Monoclonal-antibodies for the detection and quantification of the endogenous plant-growth regulator, abscisic acid. FEBS Lett 160:269-272.

52. Peuke AD, Jeschke WD, Hartung W (1994) The uptake and flow of $C, N$ and ions betwenn roots and shoots in Ricinus-communis $L$. 3. Long-distance transport of abscisic acid depending on nitrogen nutrition and salt stress. J Exp Bot 45:741-747. 